\title{
Nuclear Fragmentation in Protontherapy
}

\author{
Patrícia Rebello and Mahir S. Hussein \\ Instituto de Física, Universidade de São Paulo, Caixa Postal 66318, CEP 05315-970, São Paulo, SP, Brazil
}

Received on 15 October, 2003

\begin{abstract}
The effect of nuclear fragmentation in the passage of $180 \mathrm{MeV}$ protons through the human body tissue is discussed. Prostate cancer protontherapy with these intermediate-energy protons is discussed in light of model calculation.
\end{abstract}

The use of heavy charged particles (hadrons) to treat cancer is an old field of research. The advantages of this therapy compared to $\mathrm{x}$-ray or $\gamma$-ray therapy are well known and will not be further discussed here $[1,2]$. We shall in this short contribution give a preliminary assessment of the nuclear fragmentation effect that accompanies $180 \mathrm{MeV}$ proton cancer therapy.

The human tissue is composed by weight of $61 \%$ oxygen, $10 \%$ hydrogen, $23 \%$ carbon and smaller percentages of other elements. When a proton traverses a certain depth of this tissue, it interacts inelastically with the atoms resulting in its energy loss. The formula that describes this loss of energy is called the Bethe-Bloch one, which exhibits a peak at almost the end of the passage distance, commonly referred to as the Bragg peak. For $180 \mathrm{MeV}$ protons the Bragg peak occurs at $x=17 \mathrm{~cm}$ inside the human tissue.

Besides the atomic energy loss, the proton induces nuclear fragmentation which generates other smaller nuclei at almost zero kinetic energy plus secondary protons of $2 / 3$ of the incident proton energy. In this short contribution we study this effect.

We use the abrasion-ablation model for the fragmentation process developed by Carlson et al.[3]. This model hinges on the idea that the fragmentation occurs in two steps: an abrasion of several nucleons or clusters followed by ablation or evaporation of the hot residues. The input into such calculation is the value of the nucleon-nucleon total cross section at the corresponding laboratory energy $\left(E_{L a b} \sim 180 \mathrm{MeV}\right)$, the density profile of the target nuclei and the energy balance that determines the temperature of the residues. The energies of the residual fragments (resulting from evaporation) can be calculated.

For this purpose we used the statistical model derived by Goldhaber [4] where the momentum distribution of projectile-fragments is explained in terms of the rms momentum of the nucleons in the original projectile.

Conservation of momenta gives (that is, $A$ target nucleons assembled with zero net three momentum)

$$
\left\langle\left[\sum_{i=1}^{A} \mathbf{p}_{\mathbf{i}}\right]^{2}\right\rangle=A\left\langle p_{i}^{2}\right\rangle+A(A-1)\left\langle\mathbf{p}_{\mathbf{i}} \cdot \mathbf{p}_{\mathbf{j}}\right\rangle=0
$$

The symbol \langle\rangle implies statistical average and it is assumed that the momenta are distributed randomly (Fermi motion).

Accordingly,

$$
\left\langle\mathbf{p}_{\mathbf{i}} \cdot \mathbf{p}_{\mathbf{j}}\right\rangle=-\frac{1}{(A-1)}\left\langle p_{i}^{2}\right\rangle
$$

We know that for a Fermi gas $\left\langle p_{i}^{2}\right\rangle=\frac{3}{5} p_{\text {Fermi }}^{2}$ with $p_{F e r m i}$ being the Fermi momentum given roughly by $p_{\text {Fermi }}=230 \mathrm{MeV} / \mathrm{c}$.

If a fragment of mass $F$ is emitted from the nucleus $A$, then the momentum distribution is described by a Gaussian function with a width $\sigma^{2}$ (the momentum dispersion of a $F$ mass fragment) as:

$$
\sigma^{2}=\left\langle\left[\sum_{i=1}^{F} \mathbf{p}_{\mathbf{i}}\right]^{2}\right\rangle=F\left\langle p_{i}^{2}\right\rangle+F(F-1)\left\langle\mathbf{p}_{\mathbf{i}} \cdot \mathbf{p}_{\mathbf{j}}\right\rangle
$$

which using eq. (2) gives:

$$
\sigma^{2}=\frac{F(A-F)}{A-1}\left\langle p_{i}^{2}\right\rangle
$$

The energy range of the internal motion of fragment $F$ is simply:

$$
E_{F} \simeq \frac{\sigma^{2}}{2 m_{0} F}=\left(\frac{A-F}{A-1}\right) \frac{3}{5} \frac{p_{F e r m i}^{2}}{2 m_{0}}
$$

or, in $\mathrm{MeV}$ :

$$
E_{F} \simeq 14,9\left[\frac{A-F}{A-1}\right]
$$

where $A$ is the target mass and $F$ is the fragment mass.

We must observe that in a measurement of the momentum distribution of the fragment, a given Cartesian component is tagged. In this case we replace $\left\langle p_{i}^{2}\right\rangle$ in the Goldhaber formula [eq. (4)] by $\frac{\left\langle p_{i}^{2}\right\rangle}{3}$. Then $\left\langle p_{i}^{2}\right\rangle=\frac{p_{\text {Fermi }}^{2}}{5}$ which yields for eq. (6) the result (in $M e V$ ):

$$
E_{F} \simeq \frac{14.9}{3}\left[\frac{A-F}{A-1}\right]
$$


The result of the model calculation for $E=180 \mathrm{MeV}$ protons indicates that oxygen fragments mostly into:

$$
\begin{array}{cc}
\multicolumn{2}{c}{\text { Fragmentation of }{ }^{\mathbf{1 6}} \mathbf{O}} \\
\hline{ }^{15} \mathrm{O} & \sigma=42 m b \\
{ }^{15} \mathrm{~N} & \sigma=23 m b \\
{ }^{14} \mathrm{~N} & \sigma=31 m b \\
{ }^{13} \mathrm{C} & \sigma=28 m b \\
{ }^{12} \mathrm{C} & \sigma=36 m b
\end{array}
$$

In the case of the fragmentation of ${ }^{12} \mathbf{C}$, we find:

$$
\begin{array}{cc}
\frac{\text { Fragmentation of }{ }^{12} \mathbf{C}}{{ }^{11} \mathrm{C}} & \sigma=55 m b \\
{ }^{11} \mathrm{~B} & \sigma=30 m b
\end{array}
$$

All these nuclei have very small kinetic energies and may act as targets (ionized) for the impinging proton beam. Though this effect is probably quite small, a slight dispersion in the Bethe-Bloch formula may result as a consequence of secondary interactions. The detailed calculation of this effect is in progress and will be reported elsewhere.

Clearly, the human tissue is warm with an average temperature of about $310^{\circ} \mathrm{K}$. In the calculation of the reaction rate we have to take into account the thermal motion of the atoms (nuclei) through an appropriate Maxwell-Boltzmann factor. The center of mass energy of a $180 \mathrm{MeV}$ proton impinging on a $A$ mass target is

$$
E_{C M}=\frac{E_{L a b}}{1+\frac{1}{A}}=\frac{A}{A+1} E_{L a b}=\frac{A}{A+1} \frac{1}{2} m_{0} v^{2}
$$

The reaction rate is given by (for the production of species $i$ )

$$
r_{i}=n_{A} \int \sigma_{i}\left(E_{C M}\right) \exp \left(-\frac{E_{T}}{k T}\right) v d^{3} v
$$

where $v$ is the relative velocity, $v=\sqrt{2 \mu E_{C M}}$, with $\mu=$ $\frac{A}{A+1} m_{0}, \sigma_{i}\left(E_{C M}\right)$ the cross section, $n_{A}$ the density of target nuclei and $m_{0}$ is the nucleon mass $\left(m_{0} c^{2} \simeq 931 \mathrm{MeV}\right)$. The above formula for the reaction rate is quite commonly used in astrophysics to calculate the production of elements in stars as well as in the field of cold atoms where the temperature is gradually reduced to exceedingly small values. The use of a Maxwell-Boltzmann distribution for the velocity of the primary targets is a common procedure to incorporate thermal effects in such calculations.

$E_{T}$ is the kinetic energy of the target nuclei in the center of mass of the $p+A$ system. Thus $E_{T}=\frac{1}{2} \frac{A}{(A+1)^{2}} m_{0} v^{2}$ to be compared to the energy $E_{C M}$ that appears as the argument of the cross-section.

In the energy range corresponding to the Fermi motion of the fragment, the cross section may be taken constant (as a function of $E_{C M}$ ) and the resulting Gaussian integral from eq.(11) can be analytically evaluated. We find:

$$
r_{i}=2 \pi n_{A} \sigma_{i}\left\{\frac{1}{\alpha^{2}}-\frac{1}{\alpha} \exp \left(-\alpha v_{F}^{2}\right)\left[\frac{v_{F}^{2}}{2}+\frac{1}{\alpha}\right]\right\}
$$

where $\alpha=\frac{1}{2} \frac{A m_{0}}{(A+1)^{2}} \frac{1}{k T}$ and $\frac{1}{2 m_{0} F} v_{F}^{2}=E_{F}$ [given in eq.(6)] or:

$$
v_{F}^{2}=29.8 \frac{A-F}{(A-1) F m_{0}}
$$
lows:

Thus we have the expression for the reaction rate as fol-

$$
\begin{gathered}
r_{i}=2 \pi n_{A} \sigma_{i}\left\{\frac{(k T)^{2}(A+1)^{4}}{\frac{1}{4}\left(A m_{0}\right)^{2}}+\right. \\
\left.-\frac{(k T)(A+1)^{2}}{\frac{1}{2} A m_{0}} \exp \left(-\frac{A}{(A+1)^{2} F}\right)\left[\frac{k T}{2}+\frac{k T(A+1)^{2}}{\frac{1}{2} A m_{0}}\right]\right\}
\end{gathered}
$$

The temperature would influence the reaction rate by a small amount. However it is important to keep in mind the equation [11] for the assessment of the effect of possible variation of the temperature in different regions of the tissue. We are currently studying this effect.

\section{Acknowledgments}

We thank Brett Carlson for performing the fragmentation calculation. Patricia Rebello is supported by CNPq. Mahir S.Hussein is partially supported by FAPESP and CNPq.

\section{References}

[1] G. Kraft, Tumor therapy with heavy charged particles, Progress in Particle and Nuclear Physics, 2000

[2] A.J. Kreiner and A.A. Burlon, Novel Applications of Particle Accelerators to Radiotherapy, Heavy Ion Physics 16, 243 (2002).

[3] B.V. Carlson, R.C. Mastroleo and M.S. Hussein, Phys. Rev.C46, R3 (1992); B.V. Carlson, Phys. Rev. C51, 25 (1995).

[4] A.S. Goldhaber, Phys. Lett. B 53, 306 (1974). 